CRYSTALLOGRAPHIC COMMUNICATIONS

ISSN 2056-9890

Received 6 May 2021

Accepted 17 May 2021

Edited by L. Van Meervelt, Katholieke Universiteit Leuven, Belgium

Keywords: crystal structure; dihydroquinoxaline; hydrogen bond; $\pi$-stacking; $\mathrm{C}$ $\mathrm{H} \cdots \pi$ (ring).

CCDC reference: 2062956

Supporting information: this article has supporting information at journals.iucr.org/e

\section{Crystal structure and Hirshfeld surface analysis of 2-(2-oxo-3-phenyl-1,2,3,8a-tetrahydroquinoxalin-1- yl)ethyl acetate}

\author{
Nadeem Abad, ${ }^{\mathrm{a}, \mathrm{b}}$ Lhoussaine El Ghayati, ${ }^{\mathrm{c}}$ Camille Kalonji Mubengayi, ${ }^{\mathrm{d} *}$ El Mokhtar \\ Essassi, ${ }^{c}$ Savaş Kaya, ${ }^{e}$ Joel T. Mague ${ }^{\mathrm{f}}$ and Youssef Ramli ${ }^{\mathrm{a} *}$
}

\begin{abstract}
aLaboratory of Medicinal Chemistry, Drug Sciences Research Center, Faculty of Medicine and Pharmacy, Mohammed V University in Rabat, Morocco, ${ }^{\mathbf{b}}$ Department of Biochemistry, Faculty of Education \& Science, Al-Baydha University, Yemen, ' Laboratoire de Chimie Organique Heterocyclique, Faculté des Sciences, Université Mohammed V in Rabat, Morocco, ${ }^{\mathbf{d}}$ Laboratoire de Chimie et Biochimie de I'Institut Superieur des Techniques Medicales de Kinshasa, Republique Democratique du , Congo, 'Sivas Cumhuriyet University, Health Services Vocational School, Department of Pharmacy, 58140, Sivas, Turkey, and 'Department of Chemistry, Tulane University, New Orleans, LA 70118, USA.

*Correspondence e-mail: camillekalonji1@gmail.com,y.ramli@um5s.net.ma
\end{abstract}

In the title molecule, $\mathrm{C}_{18} \mathrm{H}_{16} \mathrm{~N}_{2} \mathrm{O}_{3}$, the dihydroquinoxaline moiety, with the exception of the $\mathrm{N}$ atom is essentially planar with the inner part of the methylpropanoate group $\left(\mathrm{CH}_{2}-\mathrm{CH}_{2}-\mathrm{O}\right)$ nearly perpendicular to it. In the crystal, inversion dimers formed by $\mathrm{C}-\mathrm{H} \cdots \mathrm{O}$ hydrogen bonds are connected into oblique stacks by $\pi$-stacking and $\mathrm{C}-\mathrm{H} \cdots \pi$ (ring) interactions.

\section{Chemical context}

Quinoxaline are a class of nitrogen containing heterocyclic compounds, found in many biologically active drugs (Ramli \& Essassi, 2015; Ramli et al., 2014). In addition, this heterocyclic scaffold possess anticorrosion characteristics (El Ouali et al., 2010; Zarrok et al., 2012; Tazouti et al., 2016; El Aoufir et al., 2016; Laabaissi et al., 2019). In a continuation of our recent work focused on the synthesis and biological evaluation of novel heterocyclic compounds (Guerrab et al. 2019, 2020, 2021; Abad et al., 2021a,b; Missioui et al. 2021) we report here the crystal structure of the title compound (Fig. 1). As with many biologically active molecules, the molecular conformation adopted may have a significant effect on its activity.

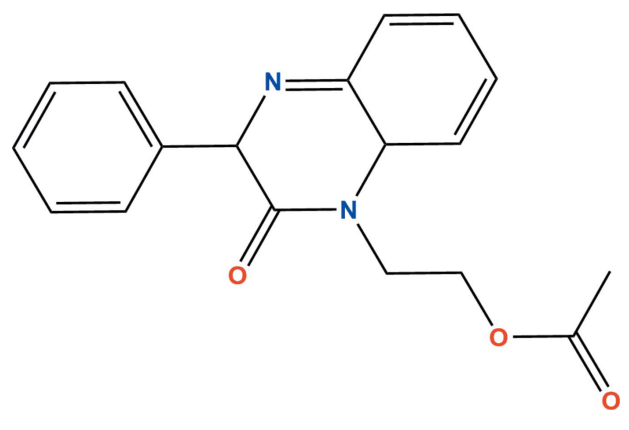

\section{Structural commentary}

The dihydroquinoxaline moiety, with the exception of $\mathrm{N} 1$, is planar to within 0.0186 (9) $\AA$ (r.m.s. deviation of the nine fitted atoms $=0.0116 \AA$ ). $\mathrm{N} 1$ lies $0.0526(12) \AA$ below the 
Table 1

Hydrogen-bond geometry $\left(\AA,^{\circ}\right)$.

$\mathrm{Cg} 2$ is the centroid of the $\mathrm{C} 1-\mathrm{C} 6$ benzene ring.

\begin{tabular}{lllll}
\hline$D-\mathrm{H} \cdots A$ & $D-\mathrm{H}$ & $\mathrm{H} \cdots A$ & $D \cdots A$ & $D-\mathrm{H} \cdots A$ \\
\hline $\mathrm{C} 4-\mathrm{H} 4 \cdots \mathrm{O} 3^{\mathrm{i}}$ & $0.974(16)$ & $2.526(16)$ & $3.4713(17)$ & $163.6(11)$ \\
$\mathrm{C} 5-\mathrm{H} 5 \cdots \mathrm{O} 3$ & $0.992(15)$ & $2.592(16)$ & $3.5435(15)$ & $160.7(12)$ \\
$\mathrm{C} 14-\mathrm{H} 14 \cdots \mathrm{O} 1$ & $0.963(15)$ & $2.232(16)$ & $2.8387(16)$ & $120.0(12)$ \\
$\mathrm{C} 16-\mathrm{H} 16 B \cdots 3^{\mathrm{ii}}$ & $0.993(14)$ & $2.553(14)$ & $3.3632(16)$ & $138.7(10)$ \\
$\mathrm{C} 18-\mathrm{H} 18 A \cdots C g 2^{\mathrm{iii}}$ & $0.97(2)$ & $2.93(2)$ & $3.7585(17)$ & $144.2(17)$ \\
\hline
\end{tabular}

Symmetry codes: (i) $\quad-x+2,-y+1,-z+1$; $\quad$ (ii) $\quad x-1, y, z$; $\quad$ (iii) $-x+1,-y+1,-z+1$.

mean plane. The C9-C14 phenyl ring is inclined to the above plane by $11.64(6)^{\circ}$ while the inner part $\left(\mathrm{CH}_{2}-\mathrm{CH}_{2}-\mathrm{O}\right)$ of the methyl propanoate substituent is nearly perpendicular to the dihydroquinoxaline unit, as indicated by the angle of $87.34(6)^{\circ}$ between the $\mathrm{N} 1 / \mathrm{C} 15 / \mathrm{C} 16 / \mathrm{O} 2$ and $\mathrm{N} 2 / \mathrm{C} 1-\mathrm{C} 8$ planes. The overall conformation is determined in part by the intra-

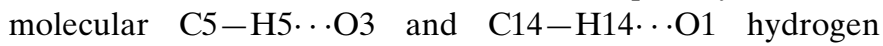
bonds (Table 1 and Fig. 1).

\section{Supramolecular features}

In the crystal, inversion dimers are formed by $\mathrm{C} 4-\mathrm{H} 4 \cdots \mathrm{O} 3^{\mathrm{i}}$ hydrogen bonds [Table 1; symmetry code: (i) $-x+2,-y+1$, $-z+1]$ and are connected into oblique stacks by a combination of $\pi$-stacking interactions between the $\mathrm{C} 1 / \mathrm{C} 6 / \mathrm{N} 1 / \mathrm{C} 7 /$ $\mathrm{C} 8 / \mathrm{N} 2$ and $\mathrm{C} 9-\mathrm{C} 14$ rings [centroid-centroid distance = 3.7786 (9) $\AA$, dihedral angle $\left.=12.20(6)^{\circ}\right]$ and in addition $\mathrm{C} 16-\mathrm{H} 16 B \cdots \mathrm{O} 3^{\mathrm{ii}}$ and $\mathrm{C} 18-\mathrm{H} 18 A \cdots C g 2^{\mathrm{iii}}$ interactions [Table 1 and Fig. 2; $C g 2$ is the centroid of the C1-C6 ring; symmetry codes: (ii) $x-1, y, z$, (iii) $-x+1,-y+1,-z+1]$. The crystal packing also shows a $\mathrm{C} 17=\mathrm{O} 3 \cdots C g 2$ interaction $[\mathrm{O} 3 \cdots C g 2=3.9578$ (12) $\AA, \quad \mathrm{C} 17 \cdots C g 2=3.7440$ (16) $\AA$, $\left.\mathrm{C} 17=\mathrm{O} 3 \cdots C g 2=71.04(8)^{\circ}\right]$.

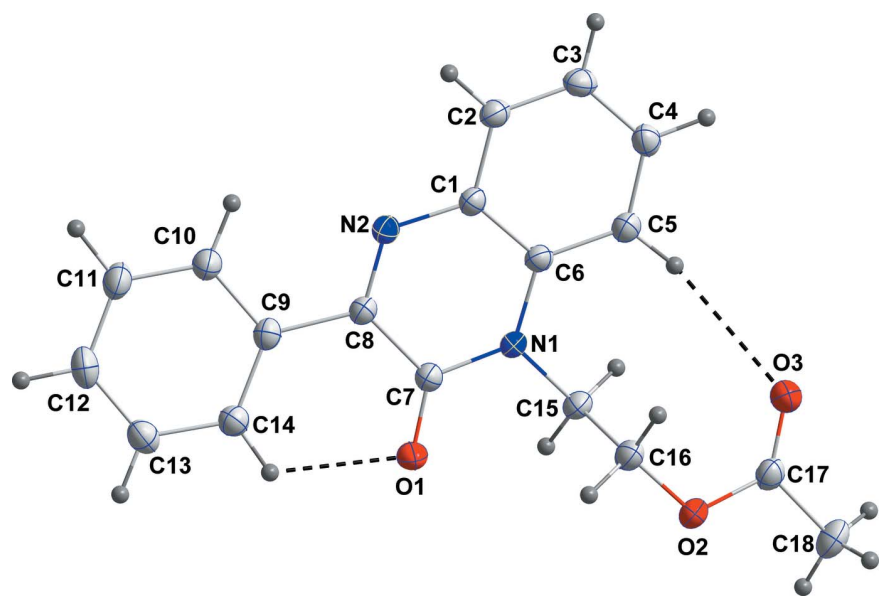

Figure 1

The title molecule with labelling scheme and $50 \%$ probability ellipsoids. The intramolecular $\mathrm{C}-\mathrm{H} \cdots \mathrm{O}$ hydrogen bonds are shown by dashed lines.

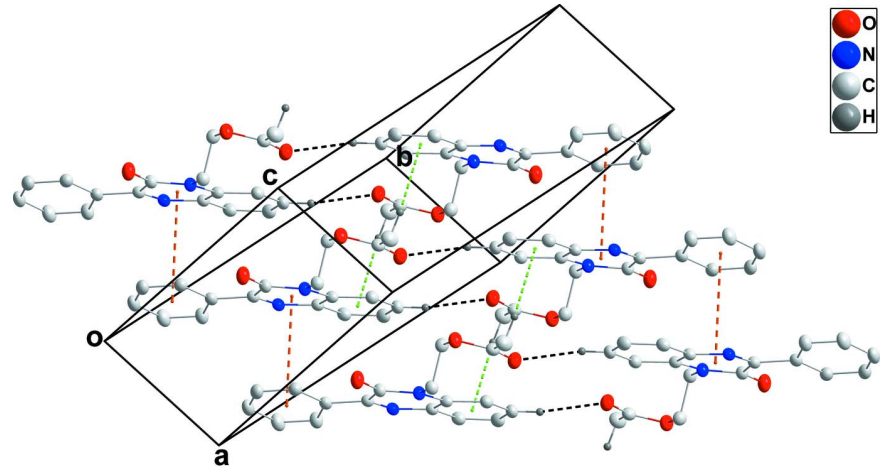

Figure 2

Perspective view of the packing. Intermolecular $\mathrm{C}-\mathrm{H} \cdots \mathrm{O}$ hydrogen bonds are shown by black dashed lines while $\pi$-stacking and $\mathrm{C}-$ $\mathrm{H} \cdots \pi$ (ring) interactions are shown, respectively, by orange and green dashed lines.

\section{Database survey}

A survey of the Cambridge Structural Database (Version 5.42, last update February 2021; Groom et al., 2016) using the search fragment II yielded 30 hits of which those most similar to the title molecule have the formula III with $R=\mathrm{Me}$ and $R^{\prime}=$ $\mathrm{CH}_{2} \mathrm{CO}_{2} \mathrm{H}$ (DEZJAW; Missioui et al., 2018), $\mathrm{CH}_{2} \mathrm{C} \equiv \mathrm{CH}$ (DUCYUW; Benzeid et al., 2009a), benzyl [DUSHUV (Ramli et al., 2010b) and DUSHUV01 (Ramli et al., 2018)], Et (IGANOU; Benzeid et al., 2008), $\mathrm{CH}_{2} \mathrm{CH}=\mathrm{CH}_{2}$ (YUPXAJ; Ramli et al., 2010a), with $R=\mathrm{CF}_{3}$ and $R^{\prime}=i$-Bu (DUBPUO; Wei et al., 2019), with $R=\mathrm{Ph}$ and $R^{\prime}=\mathrm{CH}_{2}\left(\right.$ cyclo $\left.-\mathrm{CHCH}_{2} \mathrm{O}\right)$ (NIBXEE; Abad et al., 2018a), benzyl (PUGGII; Benzeid et al., 2009b), $\mathrm{CH}_{2} \mathrm{CH}_{2} \mathrm{CH}_{2} \mathrm{OH}$ (RIRBOM; Abad et al., 2018b), $\mathrm{CH}_{2} \mathrm{CO}_{2} \mathrm{Et}$ (XEXWIJ; Abad et al., 2018c), $\mathrm{CH}_{2} \mathrm{CH}=\mathrm{CH}_{2}$ (YAJGEX; Benzeid et al., 2011) and with $R=3-\mathrm{NO}_{2}-\mathrm{C}_{6} \mathrm{H}_{4}$ and $R^{\prime}=$ benzyl (XIKHAD; Das et al., 2018).<smiles>CCn1c(=O)c(C)nc2ccccc21</smiles>

II<smiles>[R]c1nc2ccccc2n([R])c1=O</smiles>

III
In the majority of the hits, the dihydroquinoxaline ring is essentially planar with the dihedral angle between the constituent rings being less than $1^{\circ}$ or having the nitrogen bearing the exocyclic substituent less than $0.03 \AA$ from the mean plane of the remaining nine atoms. Two notable exceptions are DEZJAW, where the dihedral angle between the two rings is $3.32^{\circ}$, and RIRBOM, where the nitrogen bearing the exocyclic substituent deviates by $0.062 \AA$ from the plane defined by the other nine atoms.

\section{Hirshfeld surface analysis}

An effective means of probing intermolecular interactions is Hirshfeld surface analysis (McKinnon et al., 2007; Spackman 
(a)

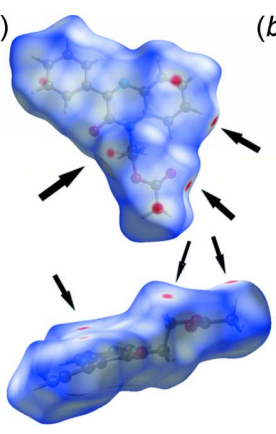

(b)
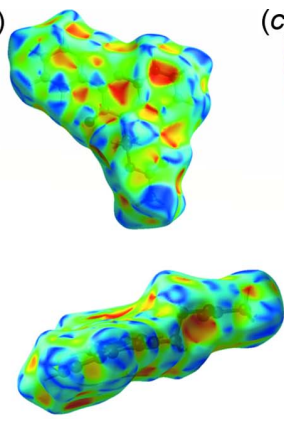

(c)

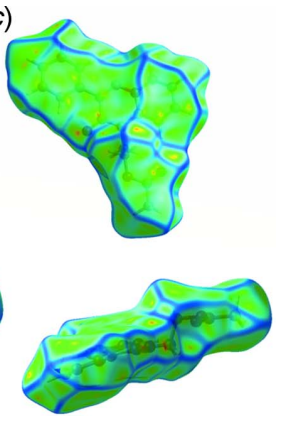

Figure 3

Front (top) and side (bottom) views of the Hirshfeld surface plotted over (a) $d_{\text {norm }},(b)$ shape-index and (c) curvature.

\& Jayatilaka, 2009), which can be conveniently carried out with Crystal Explorer 17 (Turner et al., 2017). A detailed description of the use of Crystal Explorer 17 and the plots obtained has been published (Tan et al., 2019) and will not be given here. Fig. $3 a$ presents front (top) and side (bottom) views of the Hirshfeld surface plotted over $d_{\text {norm }}$ in the range -0.1367 to 1.2965 a.u. One of the intramolecular $\mathrm{C}-\mathrm{H} \cdots \mathrm{O}$ hydrogen bonds is indicated by the arrow at the left in the front view while those leading to the formation of the inversion dimers are shown by the arrows on the right of the front view. The $\mathrm{C}-\mathrm{H} \cdots \pi$ (ring) interaction and the $\pi$-stacking interactions are represented by the red spots designated by arrows in the side view. Fig. $3 b$ presents the same two views of the surface plotted over the shape-index. In the front view, the $\pi$-stacking interaction is evident at the center as an orange triangle surrounded by blue triangles. Fig. $3 c$ has the same two views of the surface plotted over the curvature index, with the flat area in the center indicating the locus of the $\pi$-stacking interaction. Fig. 4 presents fingerprint plots for all intermolecular interactions $(a)$ and those delineated into $\mathrm{H} \cdots \mathrm{H}$ contacts $(b, 49.4 \%), \mathrm{H} \cdots \mathrm{O} / \mathrm{O} \cdots \mathrm{H}$ contacts $(c, 18.2 \%), \mathrm{H} \cdots \mathrm{C} /$ $\mathrm{C} \cdots \mathrm{H}$ contacts $(d, 17.8 \%)$ and $\mathrm{C} \cdots \mathrm{C}$ contacts $(e, 7.2 \%)$.

\section{Synthesis and crystallization}

To a solution of 2-oxo-3-phenyl-1,2-dihydroquinoxaline ( $0.5 \mathrm{~g}$, $2.25 \mathrm{mmol})$ in $N, N$-dimethylformamide $(15 \mathrm{ml})$ were added 2-bromoethyl acetate $(0.4 \mathrm{ml}, 2.25 \mathrm{mmol})$, potassium carbonate $(0.31 \mathrm{~g}, 2.25 \mathrm{mmol})$ and a catalytic quantity of tetra- $n$ butylammonium bromide. The reaction mixture was stirred at room temperature for $24 \mathrm{~h}$. The solution was filtered and the solvent removed under reduced pressure. The residue thus obtained was chromatographed on a silica gel column using a hexane/ethyl acetate 9.5: 0.5 mixture as eluent. The solid obtained was recrystallized from ethanol solution to afford colorless column-like specimen of the title compound. Yield: 0.50 g, $67 \%$; m.p. 471-473 K.

${ }^{1} \mathrm{H}$ NMR (Bruker Avance $\left.300 \mathrm{MHz}, \mathrm{CDCl}_{3}\right) \delta(\mathrm{ppm}): 8.24$ $(d, 2 \mathrm{H}, \mathrm{Ar}-\mathrm{H}) ; 7.91(d, 1 \mathrm{H}, \mathrm{Ar}-\mathrm{H}) ; 7.82(m, 3 \mathrm{H}, \mathrm{Ar}-\mathrm{H})$; $7.53(m, 1 \mathrm{H}, \mathrm{Ar}-\mathrm{H}) ; 7.25(m, 2 \mathrm{H}, \mathrm{Ar}-\mathrm{H}) ; 4.73(t, 2 \mathrm{H}, \mathrm{O}-$ $\left.\mathrm{CH}_{2}\right) ; 3.92\left(t, 2 \mathrm{H}, \mathrm{N}-\mathrm{CH}_{2}\right) ; 2.23\left(s, 3 \mathrm{H}, \mathrm{OCOCH}_{3}\right)$.
${ }^{13} \mathrm{C}$ NMR (Bruker Avance $75 \mathrm{MHz}, \mathrm{CDCl}_{3}$ ) $\delta(\mathrm{ppm}): 46.15$ $\left(\mathrm{N}-\mathrm{CH}_{2}\right) ; 61.15\left(\mathrm{O}-\mathrm{CH}_{2}\right) ; 114.38,123.82,127.01,127.72$, $128.13,128.96,129.68,130.33,130.45,130.62(\mathrm{CH}-\mathrm{Ar}) ; 132.78$, 133.36, 135.40, 136.05, 154.24(Cq); $156.92(\mathrm{C}=\mathrm{O}) ; 177.82(\mathrm{O}-$ $\mathrm{C}=\mathrm{O}$ ).

\section{Refinement}

Crystal data, data collection and structure refinement details are summarized in Table 2. All hydrogen atoms were located from a difference electron-density map and freely refined.

\section{Acknowledgements}

JTM thanks Tulane University for support of the Tulane Crystallography Laboratory. Author contributions are as follows. Conceptualization, YR and EME; synthesis, NA and LEG; writing (review and editing of the manuscript) CKM, JTM and YR; formal analysis, SK and JTM; crystal-structure determination, JTM; validation, JTM and YR, project administration, YR

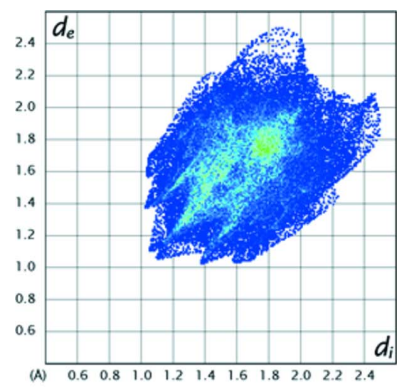

(a)

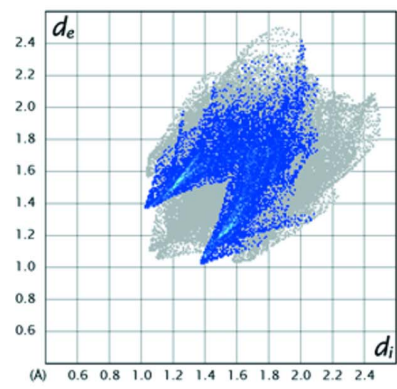

(c)

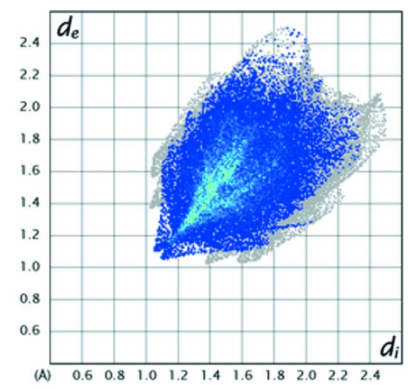

(b)

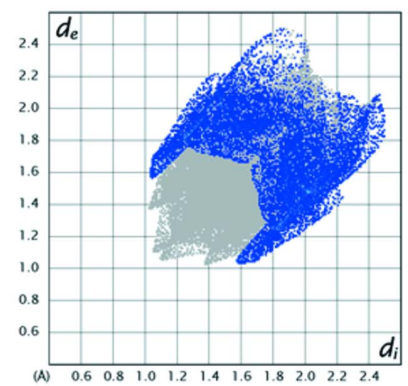

(d)

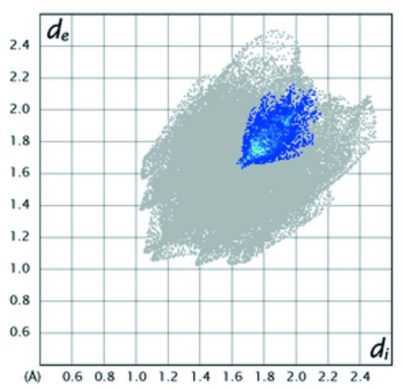

(e)

\section{Figure 4}

Two dimensional fingerprint plots showing $(a)$ all intermolecular interactions and those delineated into $(b) \mathrm{H} \cdots \mathrm{H},(c) \mathrm{H} \cdots \mathrm{O} / \mathrm{O} \cdots \mathrm{H},(d)$ $\mathrm{H} \cdots \mathrm{C} / \mathrm{C} \cdots \mathrm{H}$ and $(e) \mathrm{C} \cdots \mathrm{C}$ interactions. 
Table 2

Experimental details.

\begin{tabular}{|c|c|}
\hline \multicolumn{2}{|l|}{ Crystal data } \\
\hline Chemical formula & $\mathrm{C}_{18} \mathrm{H}_{16} \mathrm{~N}_{2} \mathrm{O}_{3}$ \\
\hline$M_{\mathrm{r}}$ & 308.33 \\
\hline Crystal system, space group & Triclinic, $P \overline{1}$ \\
\hline Temperature $(\mathrm{K})$ & 120 \\
\hline$a, b, c(\AA)$ & $\begin{array}{l}5.3518(6), 11.6989(14) \\
\quad 13.3527(16)\end{array}$ \\
\hline$\alpha, \beta, \gamma\left({ }^{\circ}\right)$ & $64.019(2), 80.323(2), 76.952(2)$ \\
\hline$V\left(\AA^{3}\right)$ & $729.83(15)$ \\
\hline$Z$ & 2 \\
\hline Radiation type & Мо $K \alpha$ \\
\hline$\mu\left(\mathrm{mm}^{-1}\right)$ & 0.10 \\
\hline Crystal size $(\mathrm{mm})$ & $0.42 \times 0.18 \times 0.12$ \\
\hline \multicolumn{2}{|l|}{ Data collection } \\
\hline Diffractometer & Bruker SMART APEX CCD \\
\hline Absorption correction & $\begin{array}{l}\text { Multi-scan (SADABS; Krause et } \\
\text { al., 2015) }\end{array}$ \\
\hline$T_{\min }, T_{\max }$ & $0.87,0.99$ \\
\hline $\begin{array}{l}\text { No. of measured, independent and } \\
\text { observed }[I>2 \sigma(I)] \text { reflections }\end{array}$ & 14193, 3909, 2929 \\
\hline$R_{\text {int }}$ & 0.028 \\
\hline$(\sin \theta / \lambda)_{\max }\left(\AA^{-1}\right)$ & 0.688 \\
\hline \multicolumn{2}{|l|}{ Refinement } \\
\hline$R\left[F^{2}>2 \sigma\left(F^{2}\right)\right], w R\left(F^{2}\right), S$ & $0.049,0.142,1.00$ \\
\hline No. of reflections & 3909 \\
\hline No. of parameters & 272 \\
\hline $\mathrm{H}$-atom treatment & All $\mathrm{H}$-atom parameters refined \\
\hline$\Delta \rho_{\max }, \Delta \rho_{\min }\left(\mathrm{e} \AA^{-3}\right)$ & $0.47,-0.23$ \\
\hline
\end{tabular}

Computer programs: APEX3 and SAINT (Bruker, 2016), SHELXT (Sheldrick, 2015a), SHELXL2018/1 (Sheldrick, 2015b), DIAMOND (Brandenburg \& Putz, 2012) and SHELXTL (Sheldrick, 2008).

\section{References}

Abad, N., Ferfra, S., Essassi, E. M., Mague, J. T. \& Ramli, Y. (2021b). Z. Kristallogr. New Cryst. Struct. 236, 173-175.

Abad, N., El Bakri, Y., Sebhaoui, J., Ramli, Y., Essassi, E. M. \& Mague, J. T. (2018a). IUCrData, 3, x180610.

Abad, N., El Bakri, Y., Sebhaoui, J., Ramli, Y., Essassi, E. M. \& Mague, J. T. (2018c). IUCrData, 3, x180519.

Abad, N., Ramli, Y., Lahmidi, S., El Hafi, Y., Essassi, E. M. \& Mague, J. T. (2018b). IUCrData, 3, x181633.

Abad, N., Sallam, H. H., Al-Ostoot, F. H., Khamees, H. A., Alhoraibi, S. A., Khanum, S. A., Madegowda, M., Hafi, M. E., Mague, J. T., Essassi, E. M. \& Ramli, Y. (2021a). J. Mol. Struct. 1232, 130004.

Benzeid, H., Bouhfid, R., Massip, S., Leger, J. M. \& Essassi, E. M. (2011). Acta Cryst. E67, o2990.

Benzeid, H., Ramli, Y., Vendier, L., Essassi, E. M. \& Ng, S. W. (2009a). Acta Cryst. E65, o2196.

Benzeid, H., Saffon, N., Garrigues, B., Essassi, E. M. \& Ng, S. W. (2009b). Acta Cryst. E65, o2685.

Benzeid, H., Vendier, L., Ramli, Y., Garrigues, B. \& Essassi, E. M. (2008). Acta Cryst. E64, o2234.

Brandenburg, K. \& Putz, H. (2012). DIAMOND, Crystal Impact GbR, Bonn, Germany.
Bruker (2016). APEX3, SADABS and SAINT. Bruker AXS Inc., Madison, Wisconsin, USA.

Das, D. K., Pampana, V. K. K. \& Hwang, K. C. (2018). Chem. Sci. 9, 7318-7326.

El Aoufir, Y., Lgaz, H., Bourazmi, H., Kerroum, Y., Ramli, Y., Guenbour, A., Salghi, R., El-Hajjaji, F., Hammouti, B. \& Oudda, H. (2016). J. Mater. Environ. Sci. 7, 4330-4347.

El Ouali, I., Hammouti, B., Aouniti, A., Ramli, Y., Azougagh, M., Essassi, E. M. \& Bouachrine, M. (2010). J. Mater. Envir. Sci. 1, 1-8.

Groom, C. R., Bruno, I. J., Lightfoot, M. P. \& Ward, S. C. (2016). Acta Cryst. B72, 171-179.

Guerrab, W., Chung, I.-M., Kansiz, S., Mague, J. T., Dege, N., Taoufik, J., Salghi, R., Ali, I. H., Chung, I. M., Lgaz, H. \& Ramli, Y. (2019). J. Mol. Struct. 1197, 127630.

Guerrab, W., Lgaz, H., Kansiz, S., Mague, J. T., Dege, N., Ansar, M., Marzouki, R., Taoufik, J., Ali, I. H., Chung, I. M. \& Ramli, Y. (2020). J. Mol. Struct. 1205, 127630.

Guerrab, W., Missioui, M., Zaoui, Y., Mague, J. T. \& Ramli, Y. (2021). Z. Kristallogr. New Cryst. Struct. 236, 133-134.

Krause, L., Herbst-Irmer, R., Sheldrick, G. M. \& Stalke, D. (2015). J. Appl. Cryst. 48, 3-10.

Laabaissi, T., Benhiba, F., Rouifi, Z., Allali, M., Missioui, M., Ourrak, K., Oudda, H., Ramli, Y., Warad, I. \& Zarrouk, A. (2019). Int. J. Corros. Scale Inhib. 8, 241-256.

McKinnon, J. J., Jayatilaka, D. \& Spackman, M. A. (2007). Chem. Commun. pp. 3814-3816.

Missioui, M., El Fal, M., Taoufik, J., Essassi, E. M., Mague, J. T. \& Ramli, Y. (2018). IUCRData 3, x180882.

Missioui, M., Mortada, S., Guerrab, W., Serdaroğlu, G., Kaya, S., Mague, J. T., Essassi, E. M., Faouzi, M. E. A. \& Ramli, Y. (2021). J. Mol. Struct. In the press.

Ramli, Y., El Bakri, Y., El Ghayati, L., Essassi, E. M. \& Mague, J. T. (2018). IUCrData 3, x180390.

Ramli, Y. \& Essassi, E. M. (2015). Adv. Chem. Res. 27, 109-160.

Ramli, Y., Moussaif, A., Karrouchi, K. \& Essassi, E. M. (2014). J. Chem. Article ID 563406, 1-21.

Ramli, Y., Moussaif, A., Zouihri, H., Lazar, S. \& Essassi, E. M. (2010b). Acta Cryst. E66, o1922.

Ramli, Y., Slimani, R., Zouihri, H., Lazar, S. \& Essassi, E. M. (2010a). Acta Cryst. E66, o1767.

Sheldrick, G. M. (2008). Acta Cryst. A64, 112-122.

Sheldrick, G. M. (2015a). Acta Cryst. A71, 3-8.

Sheldrick, G. M. (2015b). Acta Cryst. C71, 3-8.

Spackman, M. A. \& Jayatilaka, D. (2009). CrystEngComm, 11, 19-32.

Tan, S. L., Jotani, M. M. \& Tiekink, E. R. T. (2019). Acta Cryst. E75, 308-318.

Tazouti, A., Galai, M., Touir, R., Touhami, M. E., Zarrouk, A., Ramli, Y., Saraçoğlu, M., Kaya, S., Kandemirli, F. \& Kaya, C. (2016). J. Mol. Liq. 221, 815-832.

Turner, M. J., McKinnon, J. J., Wolff, S. K., Grimwood, D. J., Spackman, P. R., Jayatilaka, D. \& Spackman, M. A. (2017). Crystal Explorer 17. The University of Western Australia.

Wei, Z., Qi, S., Xu, Y., Liu, H., Wu, J., Li, H., Xia, C. \& Duan, G. (2019). Adv. Synth. Catal. 361, 5490-5498.

Zarrok, H., Zarrouk, A., Salghi, R., Oudda, H., Hammouti, B., Ebn Touhami, M., Bouachrine, M. \& Pucci, O. H. (2012). Electrochim. Acta, 30, 405-417. 


\section{supporting information}

Acta Cryst. (2021). E77, 643-646 [https://doi.org/10.1107/S2056989021005247]

Crystal structure and Hirshfeld surface analysis of 2-(2-oxo-3-phenyl-1,2,3,8atetrahydroquinoxalin-1-yl)ethyl acetate

Nadeem Abad, Lhoussaine El Ghayati, Camille Kalonji Mubengayi, El Mokhtar Essassi, Savaş

Kaya, Joel T. Mague and Youssef Ramli

Computing details

Data collection: APEX3 (Bruker, 2016); cell refinement: SAINT (Bruker, 2016); data reduction: SAINT (Bruker, 2016); program(s) used to solve structure: SHELXT (Sheldrick, 2015a); program(s) used to refine structure: SHELXL2018/1 (Sheldrick, 2015b); molecular graphics: DIAMOND (Brandenburg \& Putz, 2012); software used to prepare material for publication: SHELXTL (Sheldrick, 2008).

2-(2-Oxo-3-phenyl-1,2,3,8a-tetrahydroquinoxalin-1-yl)ethyl acetate

\section{Crystal data}

$\mathrm{C}_{18} \mathrm{H}_{16} \mathrm{~N}_{2} \mathrm{O}_{3}$

$M_{r}=308.33$

Triclinic, $P \overline{1}$

$a=5.3518(6) \AA$

$b=11.6989(14) \AA$

$c=13.3527(16) \AA$

$\alpha=64.019(2)^{\circ}$

$\beta=80.323(2)^{\circ}$

$\gamma=76.952(2)^{\circ}$

$V=729.83(15) \AA^{3}$

\section{Data collection}

Bruker SMART APEX CCD diffractometer

Radiation source: fine-focus sealed tube Graphite monochromator

Detector resolution: 8.3333 pixels $\mathrm{mm}^{-1}$

$\varphi$ and $\omega$ scans

Absorption correction: multi-scan

(SADABS; Krause et al., 2015)

$T_{\min }=0.87, T_{\max }=0.99$

Refinement

Refinement on $F^{2}$

Least-squares matrix: full

$R\left[F^{2}>2 \sigma\left(F^{2}\right)\right]=0.049$

$w R\left(F^{2}\right)=0.142$

$S=1.00$

3909 reflections
$Z=2$

$F(000)=324$

$D_{\mathrm{x}}=1.403 \mathrm{Mg} \mathrm{m}^{-3}$

Mo $K \alpha$ radiation, $\lambda=0.71073 \AA$

Cell parameters from 5207 reflections

$\theta=3.1-29.3^{\circ}$

$\mu=0.10 \mathrm{~mm}^{-1}$

$T=120 \mathrm{~K}$

Column, colourless

$0.42 \times 0.18 \times 0.12 \mathrm{~mm}$

14193 measured reflections

3909 independent reflections

2929 reflections with $I>2 \sigma(I)$

$R_{\text {int }}=0.028$

$\theta_{\max }=29.3^{\circ}, \theta_{\min }=1.7^{\circ}$

$h=-7 \rightarrow 7$

$k=-16 \rightarrow 16$

$l=-18 \rightarrow 18$

272 parameters

0 restraints

Primary atom site location: structure-invariant direct methods

Secondary atom site location: difference Fourier map 
Hydrogen site location: difference Fourier map All $\mathrm{H}$-atom parameters refined $w=1 /\left[\sigma^{2}\left(F_{\mathrm{o}}^{2}\right)+(0.0999 P)^{2}\right]$

where $P=\left(F_{\mathrm{o}}^{2}+2 F_{\mathrm{c}}{ }^{2}\right) / 3$

$$
\begin{aligned}
& (\Delta / \sigma)_{\max }<0.001 \\
& \Delta \rho_{\max }=0.47 \mathrm{e} \AA^{-3} \\
& \Delta \rho_{\min }=-0.23 \mathrm{e} \AA^{-3}
\end{aligned}
$$

\section{Special details}

Experimental. The diffraction data were obtained from 3 sets of 400 frames, each of width $0.5^{\circ}$ in $\omega$, colllected at $\varphi=$ $0.00,90.00$ and $180.00^{\circ}$ and 2 sets of 800 frames, each of width $0.45^{\circ}$ in $\varphi$, collected at $\omega=-30.00$ and $210.00^{\circ}$. The scan time was $30 \mathrm{sec} /$ frame.

Geometry. All esds (except the esd in the dihedral angle between two 1.s. planes) are estimated using the full covariance matrix. The cell esds are taken into account individually in the estimation of esds in distances, angles and torsion angles; correlations between esds in cell parameters are only used when they are defined by crystal symmetry. An approximate (isotropic) treatment of cell esds is used for estimating esds involving l.s. planes.

Refinement. Refinement of $\mathrm{F}^{2}$ against ALL reflections. The weighted R-factor $\mathrm{wR}$ and goodness of fit $\mathrm{S}$ are based on $\mathrm{F}^{2}$, conventional R-factors $\mathrm{R}$ are based on $\mathrm{F}$, with $\mathrm{F}$ set to zero for negative $\mathrm{F}^{2}$. The threshold expression of $\mathrm{F}^{2}>2$ sigma( $\mathrm{F}^{2}$ ) is used only for calculating R-factors(gt) etc. and is not relevant to the choice of reflections for refinement. R-factors based on $\mathrm{F}^{2}$ are statistically about twice as large as those based on F, and R- factors based on ALL data will be even

\begin{tabular}{|c|c|c|c|c|}
\hline & $x$ & $y$ & $z$ & $U_{\text {iso }} * / U_{\mathrm{eq}}$ \\
\hline $\mathrm{O} 1$ & $0.24048(17)$ & $0.11347(8)$ & $0.45539(7)$ & $0.0287(2)$ \\
\hline $\mathrm{O} 2$ & $0.42582(17)$ & $0.24290(8)$ & $0.68691(6)$ & $0.0264(2)$ \\
\hline $\mathrm{O} 3$ & $0.74296(17)$ & $0.36037(9)$ & $0.62940(7)$ & $0.0308(2)$ \\
\hline N1 & $0.52749(18)$ & $0.24890(9)$ & $0.40271(7)$ & $0.0209(2)$ \\
\hline N2 & $0.46952(18)$ & $0.31557(9)$ & $0.18053(7)$ & $0.0205(2)$ \\
\hline $\mathrm{C} 1$ & $0.6193(2)$ & $0.37704(11)$ & $0.20843(9)$ & $0.0198(2)$ \\
\hline $\mathrm{C} 2$ & $0.7488(2)$ & $0.47088(11)$ & $0.12307(9)$ & 0.0229 \\
\hline $\mathrm{H} 2$ & $0.731(3)$ & $0.4919(13)$ & $0.0454(12)$ & $0.033(4)^{*}$ \\
\hline $\mathrm{C} 3$ & $0.9057(2)$ & $0.53135(11)$ & $0.14791(10)$ & 0.0253 \\
\hline H3 & 0.999 (3) & $0.5940(13)$ & $0.0898(11)$ & $0.025(3)^{*}$ \\
\hline $\mathrm{C} 4$ & $0.9332(2)$ & $0.50106(12)$ & $0.25984(10)$ & 0.0240 \\
\hline H4 & $1.043(3)$ & $0.5462(13)$ & $0.2757(11)$ & $0.023(3)^{*}$ \\
\hline $\mathrm{C} 5$ & $0.8070(2)$ & 0.40967 (11) & $0.34551(10)$ & 0.0229 \\
\hline H5 & $0.835(3)$ & $0.3898(15)$ & $0.4234(13)$ & $0.035(4)^{*}$ \\
\hline C6 & $0.6527(2)$ & $0.34508(11)$ & $0.32082(9)$ & $0.0197(2)$ \\
\hline $\mathrm{C} 7$ & $0.3624(2)$ & $0.19025(11)$ & $0.37929(9)$ & 0.0209 (2) \\
\hline $\mathrm{C} 8$ & $0.3496(2)$ & $0.22703(11)$ & $0.25807(9)$ & $0.0193(2)$ \\
\hline $\mathrm{C} 9$ & $0.2018(2)$ & $0.16113(11)$ & $0.22093(9)$ & $0.0210(2)$ \\
\hline $\mathrm{C} 10$ & $0.2376(3)$ & $0.18294(12)$ & $0.10782(10)$ & $0.0268(3)$ \\
\hline $\mathrm{H} 10$ & $0.365(3)$ & $0.2385(15)$ & $0.0602(13)$ & $0.040(4)^{*}$ \\
\hline C11 & $0.1007(3)$ & $0.12852(13)$ & $0.06609(10)$ & $0.0307(3)$ \\
\hline H11 & $0.131(3)$ & $0.1475(15)$ & $-0.0164(14)$ & $0.045(4)^{*}$ \\
\hline C12 & $-0.0746(3)$ & $0.05042(12)$ & $0.13563(11)$ & 0.0299 \\
\hline H12 & $-0.174(3)$ & $0.0113(13)$ & $0.1067(11)$ & $0.026(3)^{*}$ \\
\hline C13 & $-0.1069(2)$ & $0.02574(12)$ & $0.24773(11)$ & $0.0282(3)$ \\
\hline H13 & $-0.229(3)$ & $-0.0255(14)$ & $0.2925(12)$ & $0.030(4)^{*}$ \\
\hline C14 & $0.0284(2)$ & $0.08023(11)$ & $0.29077(10)$ & 0.0239 \\
\hline H14 & $-0.004(3)$ & $0.0579(14)$ & $0.3697(12)$ & $0.030(4)^{*}$ \\
\hline
\end{tabular}
larger.

Fractional atomic coordinates and isotropic or equivalent isotropic displacement parameters $\left(\AA^{2}\right)$ 


$\begin{array}{lllll}\text { C15 } & 0.5682(2) & 0.20405(12) & 0.52109(9) & 0.0224(3) \\ \text { H15A } & 0.751(3) & 0.2084(12) & 0.5233(10) & 0.019(3)^{*} \\ \text { H15B } & 0.546(2) & 0.1157(14) & 0.5606(11) & 0.023(3)^{*} \\ \text { C16 } & 0.3790(2) & 0.28659(12) & 0.57099(9) & 0.0246(3) \\ \text { H16A } & 0.388(3) & 0.3773(14) & 0.5287(11) & 0.027(3)^{*} \\ \text { H16B } & 0.200(3) & 0.2750(12) & 0.5718(10) & 0.024(3)^{*} \\ \text { C17 } & 0.6177(2) & 0.28800(12) & 0.70394(10) & 0.0246(3) \\ \text { C18 } & 0.6508(3) & 0.23944(15) & 0.82550(11) & 0.0319(3) \\ \text { H18A } & 0.545(4) & 0.305(2) & 0.8477(19) & 0.085(7)^{*} \\ \text { H18B } & 0.600(4) & 0.1579(19) & 0.8692(16) & 0.063(5)^{*} \\ \text { H18C } & 0.829(4) & 0.2289(18) & 0.8386(15) & 0.060(5)^{*} \\ \end{array}$

Atomic displacement parameters $\left(\AA^{2}\right)$

\begin{tabular}{lllllll}
\hline & $U^{11}$ & $U^{22}$ & $U^{33}$ & $U^{12}$ & $U^{13}$ & $U^{23}$ \\
\hline O1 & $0.0367(5)$ & $0.0329(5)$ & $0.0186(4)$ & $-0.0178(4)$ & $0.0010(3)$ & $-0.0080(4)$ \\
O2 & $0.0315(5)$ & $0.0335(5)$ & $0.0192(4)$ & $-0.0123(4)$ & $0.0016(3)$ & $-0.0136(4)$ \\
O3 & $0.0309(5)$ & $0.0397(5)$ & $0.0282(4)$ & $-0.0145(4)$ & $0.0022(4)$ & $-0.0175(4)$ \\
N1 & $0.0248(5)$ & $0.0241(5)$ & $0.0160(4)$ & $-0.0082(4)$ & $0.0008(4)$ & $-0.0094(4)$ \\
N2 & $0.0230(5)$ & $0.0203(5)$ & $0.0195(4)$ & $-0.0044(4)$ & $-0.0001(4)$ & $-0.0097(4)$ \\
C1 & $0.0212(5)$ & $0.0209(5)$ & $0.0188(5)$ & $-0.0040(4)$ & $0.0007(4)$ & $-0.0102(4)$ \\
C2 & $0.0267(6)$ & $0.0224(6)$ & $0.0191(5)$ & $-0.0050(5)$ & $0.0012(4)$ & $-0.0089(4)$ \\
C3 & $0.0282(6)$ & $0.0221(6)$ & $0.0236(6)$ & $-0.0081(5)$ & $0.0039(5)$ & $-0.0080(5)$ \\
C4 & $0.0239(6)$ & $0.0247(6)$ & $0.0280(6)$ & $-0.0063(5)$ & $-0.0005(4)$ & $-0.0147(5)$ \\
C5 & $0.0241(6)$ & $0.0256(6)$ & $0.0220(5)$ & $-0.0051(4)$ & $-0.0004(4)$ & $-0.0127(5)$ \\
C6 & $0.0203(5)$ & $0.0205(5)$ & $0.0185(5)$ & $-0.0043(4)$ & $0.0017(4)$ & $-0.0091(4)$ \\
C7 & $0.0237(6)$ & $0.0216(5)$ & $0.0191(5)$ & $-0.0063(4)$ & $0.0002(4)$ & $-0.0097(4)$ \\
C8 & $0.0209(5)$ & $0.0201(5)$ & $0.0178(5)$ & $-0.0029(4)$ & $-0.0013(4)$ & $-0.0092(4)$ \\
C9 & $0.0225(6)$ & $0.0200(5)$ & $0.0217(5)$ & $-0.0020(4)$ & $-0.0038(4)$ & $-0.0097(4)$ \\
C10 & $0.0338(7)$ & $0.0276(6)$ & $0.0223(5)$ & $-0.0105(5)$ & $-0.0018(5)$ & $-0.0110(5)$ \\
C11 & $0.0415(7)$ & $0.0316(7)$ & $0.0246(6)$ & $-0.0096(5)$ & $-0.0061(5)$ & $-0.0141(5)$ \\
C12 & $0.0322(7)$ & $0.0285(6)$ & $0.0373(7)$ & $-0.0059(5)$ & $-0.0088(5)$ & $-0.0188(6)$ \\
C13 & $0.0266(6)$ & $0.0269(6)$ & $0.0337(6)$ & $-0.0085(5)$ & $-0.0009(5)$ & $-0.0137(5)$ \\
C14 & $0.0235(6)$ & $0.0243(6)$ & $0.0246(6)$ & $-0.0049(4)$ & $-0.0009(4)$ & $-0.0109(5)$ \\
C15 & $0.0265(6)$ & $0.0247(6)$ & $0.0170(5)$ & $-0.0068(5)$ & $-0.0007(4)$ & $-0.0089(4)$ \\
C16 & $0.0254(6)$ & $0.0309(6)$ & $0.0202(5)$ & $-0.0069(5)$ & $-0.0011(4)$ & $-0.0122(5)$ \\
C17 & $0.0245(6)$ & $0.0294(6)$ & $0.0241(5)$ & $-0.0037(5)$ & $-0.0003(4)$ & $-0.0161(5)$ \\
C18 & $0.0388(8)$ & $0.0373(8)$ & $0.0229(6)$ & $-0.0034(6)$ & $-0.0053(5)$ & $-0.0159(6)$ \\
& & & & & & \\
& & & & & &
\end{tabular}

Geometric parameters $\left(\AA,{ }^{o}\right)$

\begin{tabular}{llll}
\hline $\mathrm{O} 1-\mathrm{C} 7$ & $1.2275(13)$ & $\mathrm{C} 9-\mathrm{C} 14$ & $1.3995(16)$ \\
$\mathrm{O} 2-\mathrm{C} 17$ & $1.3467(14)$ & $\mathrm{C} 9-\mathrm{C} 10$ & $1.4036(15)$ \\
$\mathrm{O} 2-\mathrm{C} 16$ & $1.4489(13)$ & $\mathrm{C} 10-\mathrm{C} 11$ & $1.3828(17)$ \\
$\mathrm{O} 3-\mathrm{C} 17$ & $1.2043(14)$ & $\mathrm{C} 10-\mathrm{H} 10$ & $0.990(16)$ \\
$\mathrm{N} 1-\mathrm{C} 7$ & $1.3800(14)$ & $\mathrm{C} 11-\mathrm{C} 12$ & $1.3884(19)$ \\
$\mathrm{N} 1-\mathrm{C} 6$ & $1.3882(14)$ & $\mathrm{C} 11-\mathrm{H} 11$ & $1.016(16)$ \\
$\mathrm{N} 1-\mathrm{C} 15$ & $1.4692(13)$ & $\mathrm{C} 12-\mathrm{C} 13$ & $1.3822(18)$
\end{tabular}




\begin{tabular}{|c|c|c|c|}
\hline $\mathrm{N} 2-\mathrm{C} 8$ & $1.3016(14)$ & $\mathrm{C} 12-\mathrm{H} 12$ & $0.988(14)$ \\
\hline $\mathrm{N} 2-\mathrm{C} 1$ & $1.3763(14)$ & $\mathrm{C} 13-\mathrm{C} 14$ & $1.3894(17)$ \\
\hline $\mathrm{C} 1-\mathrm{C} 2$ & $1.4022(15)$ & $\mathrm{C} 13-\mathrm{H} 13$ & $0.934(15)$ \\
\hline $\mathrm{C} 1-\mathrm{C} 6$ & $1.4114(14)$ & $\mathrm{C} 14-\mathrm{H} 14$ & $0.962(14)$ \\
\hline $\mathrm{C} 2-\mathrm{C} 3$ & $1.3721(17)$ & $\mathrm{C} 15-\mathrm{C} 16$ & $1.5155(17)$ \\
\hline $\mathrm{C} 2-\mathrm{H} 2$ & $0.972(15)$ & $\mathrm{C} 15-\mathrm{H} 15 \mathrm{~A}$ & $0.996(13)$ \\
\hline $\mathrm{C} 3-\mathrm{C} 4$ & $1.4027(16)$ & $\mathrm{C} 15-\mathrm{H} 15 \mathrm{~B}$ & $0.958(14)$ \\
\hline $\mathrm{C} 3-\mathrm{H} 3$ & $0.962(13)$ & C16-H16A & $0.967(14)$ \\
\hline $\mathrm{C} 4-\mathrm{C} 5$ & $1.3795(16)$ & C16-H16B & $0.993(14)$ \\
\hline $\mathrm{C} 4-\mathrm{H} 4$ & $0.973(14)$ & $\mathrm{C} 17-\mathrm{C} 18$ & $1.4940(16)$ \\
\hline $\mathrm{C} 5-\mathrm{C} 6$ & $1.3975(16)$ & $\mathrm{C} 18-\mathrm{H} 18 \mathrm{~A}$ & $0.97(2)$ \\
\hline $\mathrm{C} 5-\mathrm{H} 5$ & $0.992(15)$ & C18-H18B & $0.95(2)$ \\
\hline $\mathrm{C} 7-\mathrm{C} 8$ & $1.4911(14)$ & $\mathrm{C} 18-\mathrm{H} 18 \mathrm{C}$ & $0.97(2)$ \\
\hline $\mathrm{C} 8-\mathrm{C} 9$ & $1.4861(15)$ & & \\
\hline $\mathrm{C} 17-\mathrm{O} 2-\mathrm{C} 16$ & $115.33(9)$ & $\mathrm{C} 9-\mathrm{C} 10-\mathrm{H} 10$ & $117.0(9)$ \\
\hline $\mathrm{C} 7-\mathrm{N} 1-\mathrm{C} 6$ & $123.19(9)$ & $\mathrm{C} 10-\mathrm{C} 11-\mathrm{C} 12$ & $120.53(12)$ \\
\hline $\mathrm{C} 7-\mathrm{N} 1-\mathrm{C} 15$ & $116.52(9)$ & $\mathrm{C} 10-\mathrm{C} 11-\mathrm{H} 11$ & $118.3(9)$ \\
\hline $\mathrm{C} 6-\mathrm{N} 1-\mathrm{C} 15$ & $120.28(9)$ & $\mathrm{C} 12-\mathrm{C} 11-\mathrm{H} 11$ & $121.2(9)$ \\
\hline $\mathrm{C} 8-\mathrm{N} 2-\mathrm{C} 1$ & $120.49(9)$ & $\mathrm{C} 13-\mathrm{C} 12-\mathrm{C} 11$ & $119.10(11)$ \\
\hline $\mathrm{N} 2-\mathrm{C} 1-\mathrm{C} 2$ & $119.20(9)$ & $\mathrm{C} 13-\mathrm{C} 12-\mathrm{H} 12$ & $119.6(8)$ \\
\hline $\mathrm{N} 2-\mathrm{C} 1-\mathrm{C} 6$ & $121.67(10)$ & $\mathrm{C} 11-\mathrm{C} 12-\mathrm{H} 12$ & $121.3(8)$ \\
\hline $\mathrm{C} 2-\mathrm{C} 1-\mathrm{C} 6$ & $119.10(10)$ & $\mathrm{C} 12-\mathrm{C} 13-\mathrm{C} 14$ & $121.02(12)$ \\
\hline $\mathrm{C} 3-\mathrm{C} 2-\mathrm{C} 1$ & $120.73(10)$ & $\mathrm{C} 12-\mathrm{C} 13-\mathrm{H} 13$ & $117.5(9)$ \\
\hline $\mathrm{C} 3-\mathrm{C} 2-\mathrm{H} 2$ & $119.6(8)$ & $\mathrm{C} 14-\mathrm{C} 13-\mathrm{H} 13$ & $121.4(9)$ \\
\hline $\mathrm{C} 1-\mathrm{C} 2-\mathrm{H} 2$ & $119.7(8)$ & $\mathrm{C} 13-\mathrm{C} 14-\mathrm{C} 9$ & $120.32(11)$ \\
\hline $\mathrm{C} 2-\mathrm{C} 3-\mathrm{C} 4$ & $119.78(11)$ & $\mathrm{C} 13-\mathrm{C} 14-\mathrm{H} 14$ & $116.0(9)$ \\
\hline $\mathrm{C} 2-\mathrm{C} 3-\mathrm{H} 3$ & $121.2(8)$ & $\mathrm{C} 9-\mathrm{C} 14-\mathrm{H} 14$ & $123.7(9)$ \\
\hline $\mathrm{C} 4-\mathrm{C} 3-\mathrm{H} 3$ & $119.0(8)$ & $\mathrm{N} 1-\mathrm{C} 15-\mathrm{C} 16$ & $110.08(9)$ \\
\hline $\mathrm{C} 5-\mathrm{C} 4-\mathrm{C} 3$ & $120.72(11)$ & $\mathrm{N} 1-\mathrm{C} 15-\mathrm{H} 15 \mathrm{~A}$ & $106.2(7)$ \\
\hline $\mathrm{C} 5-\mathrm{C} 4-\mathrm{H} 4$ & $120.7(8)$ & $\mathrm{C} 16-\mathrm{C} 15-\mathrm{H} 15 \mathrm{~A}$ & $112.9(7)$ \\
\hline $\mathrm{C} 3-\mathrm{C} 4-\mathrm{H} 4$ & $118.5(8)$ & $\mathrm{N} 1-\mathrm{C} 15-\mathrm{H} 15 \mathrm{~B}$ & $109.3(8)$ \\
\hline $\mathrm{C} 4-\mathrm{C} 5-\mathrm{C} 6$ & $119.78(10)$ & $\mathrm{C} 16-\mathrm{C} 15-\mathrm{H} 15 \mathrm{~B}$ & $110.1(8)$ \\
\hline $\mathrm{C} 4-\mathrm{C} 5-\mathrm{H} 5$ & $118.0(9)$ & $\mathrm{H} 15 \mathrm{~A}-\mathrm{C} 15-\mathrm{H} 15 \mathrm{~B}$ & $108.2(11)$ \\
\hline C6- $65-\mathrm{H} 5$ & $122.2(9)$ & $\mathrm{O} 2-\mathrm{C} 16-\mathrm{C} 15$ & $109.30(10)$ \\
\hline $\mathrm{N} 1-\mathrm{C} 6-\mathrm{C} 5$ & $122.87(9)$ & $\mathrm{O} 2-\mathrm{C} 16-\mathrm{H} 16 \mathrm{~A}$ & $111.5(8)$ \\
\hline $\mathrm{N} 1-\mathrm{C} 6-\mathrm{C} 1$ & $117.29(10)$ & $\mathrm{C} 15-\mathrm{C} 16-\mathrm{H} 16 \mathrm{~A}$ & $111.6(8)$ \\
\hline $\mathrm{C} 5-\mathrm{C} 6-\mathrm{C} 1$ & $119.84(10)$ & $\mathrm{O} 2-\mathrm{C} 16-\mathrm{H} 16 \mathrm{~B}$ & $105.9(7)$ \\
\hline $\mathrm{O} 1-\mathrm{C} 7-\mathrm{N} 1$ & $120.36(10)$ & $\mathrm{C} 15-\mathrm{C} 16-\mathrm{H} 16 \mathrm{~B}$ & $110.3(7)$ \\
\hline $\mathrm{O} 1-\mathrm{C} 7-\mathrm{C} 8$ & $124.70(10)$ & $\mathrm{H} 16 \mathrm{~A}-\mathrm{C} 16-\mathrm{H} 16 \mathrm{~B}$ & $108.0(11)$ \\
\hline $\mathrm{N} 1-\mathrm{C} 7-\mathrm{C} 8$ & $114.94(9)$ & $\mathrm{O} 3-\mathrm{C} 17-\mathrm{O} 2$ & $123.41(10)$ \\
\hline $\mathrm{N} 2-\mathrm{C} 8-\mathrm{C} 9$ & $117.09(9)$ & $\mathrm{O} 3-\mathrm{C} 17-\mathrm{C} 18$ & $124.81(11)$ \\
\hline $\mathrm{N} 2-\mathrm{C} 8-\mathrm{C} 7$ & $122.12(10)$ & $\mathrm{O} 2-\mathrm{C} 17-\mathrm{C} 18$ & $111.77(10)$ \\
\hline $\mathrm{C} 9-\mathrm{C} 8-\mathrm{C} 7$ & $120.78(9)$ & $\mathrm{C} 17-\mathrm{C} 18-\mathrm{H} 18 \mathrm{~A}$ & $104.9(13)$ \\
\hline $\mathrm{C} 14-\mathrm{C} 9-\mathrm{C} 10$ & $118.14(10)$ & $\mathrm{C} 17-\mathrm{C} 18-\mathrm{H} 18 \mathrm{~B}$ & $113.1(11)$ \\
\hline $\mathrm{C} 14-\mathrm{C} 9-\mathrm{C} 8$ & $124.43(10)$ & $\mathrm{H} 18 \mathrm{~A}-\mathrm{C} 18-\mathrm{H} 18 \mathrm{~B}$ & $110.9(18)$ \\
\hline $\mathrm{C} 10-\mathrm{C} 9-\mathrm{C} 8$ & $117.43(10)$ & $\mathrm{C} 17-\mathrm{C} 18-\mathrm{H} 18 \mathrm{C}$ & $111.5(11)$ \\
\hline $\mathrm{C} 11-\mathrm{C} 10-\mathrm{C} 9$ & $120.87(11)$ & $\mathrm{H} 18 \mathrm{~A}-\mathrm{C} 18-\mathrm{H} 18 \mathrm{C}$ & $110.2(17)$ \\
\hline
\end{tabular}




$\begin{array}{llll}\mathrm{C} 11-\mathrm{C} 10-\mathrm{H} 10 & 122.2(9) & \mathrm{H} 18 \mathrm{~B}-\mathrm{C} 18-\mathrm{H} 18 \mathrm{C} & 106.3(16) \\ \mathrm{C} 8-\mathrm{N} 2-\mathrm{C} 1-\mathrm{C} 2 & 179.20(10) & \mathrm{O} 1-\mathrm{C} 7-\mathrm{C} 8-\mathrm{N} 2 & 175.20(11) \\ \mathrm{C} 8-\mathrm{N} 2-\mathrm{C} 1-\mathrm{C} 6 & 1.29(17) & \mathrm{N} 1-\mathrm{C} 7-\mathrm{C} 8-\mathrm{N} 2 & -5.32(16) \\ \mathrm{N} 2-\mathrm{C} 1-\mathrm{C} 2-\mathrm{C} 3 & -178.09(10) & \mathrm{O} 1-\mathrm{C} 7-\mathrm{C} 8-\mathrm{C} 9 & -6.07(18) \\ \mathrm{C} 6-\mathrm{C} 1-\mathrm{C} 2-\mathrm{C} 3 & -0.13(17) & \mathrm{N} 1-\mathrm{C} 7-\mathrm{C} 8-\mathrm{C} 9 & 173.42(9) \\ \mathrm{C} 1-\mathrm{C} 2-\mathrm{C} 3-\mathrm{C} 4 & -1.29(18) & \mathrm{N} 2-\mathrm{C} 8-\mathrm{C} 9-\mathrm{C} 14 & -168.77(10) \\ \mathrm{C} 2-\mathrm{C} 3-\mathrm{C} 4-\mathrm{C} 5 & 0.91(18) & \mathrm{C} 7-\mathrm{C} 8-\mathrm{C} 9-\mathrm{C} 14 & 12.44(17) \\ \mathrm{C} 3-\mathrm{C} 4-\mathrm{C} 5-\mathrm{C} 6 & 0.92(18) & \mathrm{N} 2-\mathrm{C} 8-\mathrm{C} 9-\mathrm{C} 10 & 10.61(16) \\ \mathrm{C} 7-\mathrm{N} 1-\mathrm{C} 6-\mathrm{C} 5 & 175.87(10) & \mathrm{C} 7-\mathrm{C} 8-\mathrm{C} 9-\mathrm{C} 10 & -168.19(10) \\ \mathrm{C} 15-\mathrm{N} 1-\mathrm{C} 6-\mathrm{C} 5 & -4.42(16) & \mathrm{C} 14-\mathrm{C} 9-\mathrm{C} 10-\mathrm{C} 11 & 1.58(18) \\ \mathrm{C} 7-\mathrm{N} 1-\mathrm{C} 6-\mathrm{C} 1 & -4.13(16) & \mathrm{C} 8-\mathrm{C} 9-\mathrm{C} 10-\mathrm{C} 11 & -177.84(11) \\ \mathrm{C} 15-\mathrm{N} 1-\mathrm{C} 6-\mathrm{C} 1 & 175.58(10) & \mathrm{C} 9-\mathrm{C} 10-\mathrm{C} 11-\mathrm{C} 12 & -0.4(2) \\ \mathrm{C} 4-\mathrm{C} 5-\mathrm{C} 6-\mathrm{N} 1 & 177.66(10) & \mathrm{C} 10-\mathrm{C} 11-\mathrm{C} 12-\mathrm{C} 13 & -1.2(2) \\ \mathrm{C} 4-\mathrm{C} 5-\mathrm{C} 6-\mathrm{C} 1 & -2.34(17) & \mathrm{C} 11-\mathrm{C} 12-\mathrm{C} 13-\mathrm{C} 14 & 1.52(19) \\ \mathrm{N} 2-\mathrm{C} 1-\mathrm{C} 6-\mathrm{N} 1 & -0.14(16) & \mathrm{C} 12-\mathrm{C} 13-\mathrm{C} 14-\mathrm{C} 9 & -0.28(19) \\ \mathrm{C} 2-\mathrm{C} 1-\mathrm{C} 6-\mathrm{N} 1 & -178.05(10) & \mathrm{C} 10-\mathrm{C} 9-\mathrm{C} 14-\mathrm{C} 13 & -1.26(17) \\ \mathrm{N} 2-\mathrm{C} 1-\mathrm{C} 6-\mathrm{C} 5 & 179.86(10) & \mathrm{C} 8-\mathrm{C} 9-\mathrm{C} 14-\mathrm{C} 13 & 178.11(10) \\ \mathrm{C} 2-\mathrm{C} 1-\mathrm{C} 6-\mathrm{C} 5 & 1.95(16) & \mathrm{C} 7-\mathrm{N} 1-\mathrm{C} 15-\mathrm{C} 16 & -92.28(12) \\ \mathrm{C} 6-\mathrm{N} 1-\mathrm{C} 7-\mathrm{O} 1 & -173.92(10) & \mathrm{C} 6-\mathrm{N} 1-\mathrm{C} 15-\mathrm{C} 16 & 88.00(12) \\ \mathrm{C} 15-\mathrm{N} 1-\mathrm{C} 7-\mathrm{O} 1 & 6.36(16) & \mathrm{C} 17-\mathrm{O} 2-\mathrm{C} 16-\mathrm{C} 15 & 82.16(12) \\ \mathrm{C} 6-\mathrm{N} 1-\mathrm{C} 7-\mathrm{C} 8 & 6.57(15) & \mathrm{N} 1-\mathrm{C} 15-\mathrm{C} 16-\mathrm{O} 2 & -178.70(9) \\ \mathrm{C} 15-\mathrm{N} 1-\mathrm{C} 7-\mathrm{C} 8 & -173.15(9) & \mathrm{C} 16-\mathrm{O} 2-\mathrm{C} 17-\mathrm{O} 3 & 0.52(16) \\ \mathrm{C} 1-\mathrm{N} 2-\mathrm{C} 8-\mathrm{C} 9 & -177.24(9) & \mathrm{C} 16-\mathrm{O} 2-\mathrm{C} 17-\mathrm{C} 18 & 179.37(10) \\ \mathrm{C} 1-\mathrm{N} 2-\mathrm{C} 8-\mathrm{C} 7 & 1.54(17) & & \\ & & & \end{array}$

Hydrogen-bond geometry $\left(A,{ }^{\circ}\right)$

$\mathrm{Cg} 2$ is the centroid of the $\mathrm{C} 1-\mathrm{C} 6$ benzene ring.

\begin{tabular}{lllll}
\hline$D-\mathrm{H} \cdots A$ & $D-\mathrm{H}$ & $\mathrm{H} \cdots A$ & $D \cdots A$ & $D-\mathrm{H} \cdots A$ \\
\hline $\mathrm{C} 4-\mathrm{H} 4 \cdots \mathrm{O} 3^{\mathrm{i}}$ & $0.974(16)$ & $2.526(16)$ & $3.4713(17)$ & $163.6(11)$ \\
$\mathrm{C} 5-\mathrm{H} 5 \cdots \mathrm{O} 3$ & $0.992(15)$ & $2.592(16)$ & $3.5435(15)$ & $160.7(12)$ \\
$\mathrm{C} 14-\mathrm{H} 14 \cdots \mathrm{O} 1$ & $0.963(15)$ & $2.232(16)$ & $2.8387(16)$ & $120.0(12)$ \\
$\mathrm{C} 16-\mathrm{H} 16 B \cdots \mathrm{O} 3^{\mathrm{ii}}$ & $0.993(14)$ & $2.553(14)$ & $3.3632(16)$ & $138.7(10)$ \\
$\mathrm{C} 18-\mathrm{H} 18 A \cdots C g 2^{\mathrm{iii}}$ & $0.97(2)$ & $2.93(2)$ & $3.7585(17)$ & $144.2(17)$
\end{tabular}

Symmetry codes: (i) $-x+2,-y+1,-z+1$; (ii) $x-1, y, z$; (iii) $-x+1,-y+1,-z+1$. 